\title{
Lymphogranuloma venereum in the differential diagnosis of proctitis
}

Background and epidemiology: Lymphogranuloma venereum (LGV) is a systemic STD caused by infection by Chlamydia trachomatis serotypes L1, L2 and L3, which are endemic in parts of Africa, Asia, South America and the Caribbean but rare in Western countries. Unlike the more common $C$. trachomatis serotypes $\mathrm{A}-\mathrm{K}$, which tend to be confined to columnar epithelial cells in the genital tract and eye, LGV serotypes predominantly infect monocytes and macrophages and pass through the epithelial surface to regional lymph nodes. ${ }^{1}$

The clinical course of LGV can be divided into 3 stages: inoculation, regional lymph node invasion, and late sequelae involving the genitals or rectum or both. The primary stage presents with a small, painless papule, typically on the penis, vulva or rectum, after an incubation period of 3-30 days. The lesions may not be noticed by infected people. The second stage usually presents some weeks later with painful, unilateral lymph nodes in the inguinal or anorectal region. Since lymph vessels from the cervix and vagina tend to drain to the retroperitoneal area, proctitis is a common presentation of LGV in women, whereas men present more often with unilateral, enlarged, painful inguinal nodes. Men who engage in anal sex with other men more commonly present with proctitis, which results from direct inoculation in the anal region. Chronic untreated LGV, the third stage, can lead to lymphatic obstruction, resulting in elephantitis of the genitalia in either sex; rectal involvement can lead to the formation of stric- tures and fistulas, which may require surgery to correct. ${ }^{1}$

Although LGV is rare in industrialized countries, a recent large outbreak (92 cases over 17 months) in the Netherlands among men who have sex with men prompted the US Centers for Disease Control and Prevention to alert health care providers in industrialized countries about the possibility and implications of such an outbreak. ${ }^{2}$ Preliminary evaluation of the outbreak determined that all of the patients were white, concurrent STDs were prevalent, and the majority of patients were HIV-positive and had participated in casual sex gatherings (e.g., leather scene parties) and had had unprotected anal sex within the 12 months before onset of symptoms. Only 1 patient presented with the classic symptoms of LGV (inguinal adenopathy and genital ulcer). Most of the infected men presented with gastrointestinal symptoms (bloody proctitis with anal discharge and constipation). In all cases, LGV was associated with the presence of high-titre $C$. trachomatis antibodies in sera. ${ }^{2}$

Clinical management: The differential diagnosis of sexually acquired ulceration with or without accompanying inguinal adenopathy includes chancroid, herpes, syphilis and donovanosis (granuloma inguinale). ${ }^{1}$ The diagnosis of LGV is made primarily on the basis of clinical findings. Serologic tests for C. trachomatis (microimmunofluorescence or complement fixation) can support the diagnosis, as can detection of the pathogen in bubo fluid by either culture or nucleic acid testing. ${ }^{3}$
Fluctuant nodes should be aspirated through healthy skin. Incision and drainage or excision of nodes may delay healing.

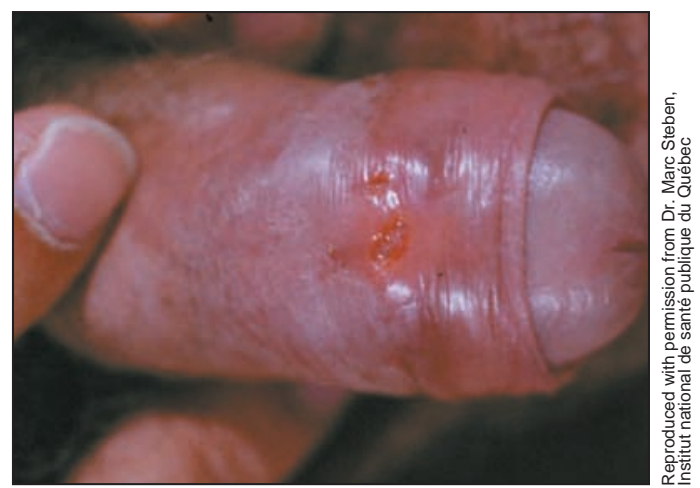

Recommended regimens of treatment of LGV include $100 \mathrm{mg}$ of doxycycline taken orally twice a day for 14-21 days, or $50 \mathrm{mg}$ of erythromycin taken orally 4 times a day for 14-21 days. Sex partners who had contact with the patient within 30 days of the patient's onset of symptoms should be given either $1 \mathrm{~g}$ of azithromycin in a single dose or $100 \mathrm{mg}$ of doxycycline twice daily for 7 days.,

Prevention: Barrier methods, such as condoms, provide good protection against transmission.

\section{Erica Weir}

CMAF

\section{References}

1. Mabey D, Peeling RW. Lymphogranuloma venereum. Sex Transm Infect 2002;78:90-2. Available: http://sti. bmijournals.com/cgi/content/full/78/ 2/90 (accessed 2004 Dec 2).

2. Lymphogranuloma venereum among men who have sex with men Netherlands, 2003-2004. MMWR Morb Mortal Wkly Rep 2004;53:985-8.

3. Guidelines for the management of sexually transmitted infections. Geneva: World Health Organization; 2003. Available: www.who.int/reproductivehealth/publications/rhr_01_10.index. html (accessed 2004 Dec 2). 